Meta

Journal des traducteurs

Translators' Journal

\title{
Konstruktive Evaluation: Versuch eines Evaluationskonzepts für den Unterricht
}

\section{Alain Rosenmund}

Volume 46, numéro 2, juin 2001

Évaluation : paramètres, méthodes, aspects pédagogiques / Evaluation: Parameters, Methods, Pedagogical Aspects

URI : https://id.erudit.org/iderudit/003987ar

DOI : https://doi.org/10.7202/003987ar

Aller au sommaire du numéro

Éditeur(s)

Les Presses de l'Université de Montréal

ISSN

0026-0452 (imprimé)

1492-1421 (numérique)

Découvrir la revue

Citer cet article

Rosenmund, A. (2001). Konstruktive Evaluation: Versuch eines Evaluationskonzepts für den Unterricht. Meta, 46(2), 301-310. https://doi.org/10.7202/003987ar
Résumé de l'article

Partant du constat qu'aucune traduction ne saurait être évaluée hors contexte et sans référence à sa finalité, afin d'augmenter le degré d'objectivité de l'évaluation et de préparer l'étudiant à l'environnement professionnel, il est proposé d'apprécier les traductions entreprises par les étudiants pendant l'année scolaire en fonction du cahier des charges établi préalablement par le professeur et les étudiants. 


\title{
Konstruktive Evaluation: \\ Versuch eines Evaluationskonzepts \\ für den Unterricht
}

\author{
ALAIN ROSENMUND \\ École de Traduction et d'Interprétation de Genève, Suisse
}

\begin{abstract}
RÉSUMÉ
Partant du constat qu'aucune traduction ne saurait être évaluée hors contexte et sans référence à sa finalité, afin d'augmenter le degré d'objectivité de l'évaluation et de préparer l'étudiant à l'environnement professionnel, il est proposé d'apprécier les traductions entreprises par les étudiants pendant l'année scolaire en fonction du cahier des charges établi préalablement par le professeur et les étudiants.
\end{abstract}

\begin{abstract}
All translations should be assessed with regard to their context and aim. Therefore, and in order to objectivize the assessment as well as to prepare the students for the professional environment, the assessment of students' translations should be based on specifications which have been worked out by the professor or lecturer and students beforehand.
\end{abstract}

MOTS-CLÉS/KEYWORDS

formation professionnelle en traduction, évaluation de la traduction, qualité de la traduction, objectivité, contexte

\section{Einleitung}

Das Angebot, Übersetzungen parallel zum Übersetzungsunterricht in schriftlicher Form abgeben zu können, und diese Arbeiten darauf korrigiert zurückzubekommen, wird der Student (die weibliche Form ist immer mitgemeint) im Allgemeinen gerne annehmen, da dieses Feedback für ihn eine Möglichkeit darstellt, seine Kompetenzen auszubauen und Fortschritte, die er gemacht hat, zu messen. Deshalb lohnt es, sich Gedanken darüber zu machen, wie mögliche Evaluationskonzepte im Rahmen des (Hochschul-)Unterrichts aussehen könnten. Bei diesem Artikel richtet sich das $\mathrm{Au}-$ genmerk auf die Evaluation von Übersetzungen, die während des Semesters geschrieben werden. Auf den Sonderfall, den die Bewertung von Klausuren darstellt, wird nicht eingegangen. Dies, weil bei der Klausur die Lernkontrolle in Hinblick auf den Lehrplan, die Zensur und allenfalls darauf folgende Sanktionen im Vordergrund stehen. Bei der Übersetzung hingegen, die während des Semesters geschrieben wird, und bei deren Evaluation, wird dem pädagogischen Aspekt mehr Gewicht beigemessen. Dieser Artikel will versuchen, ein Evaluationskonzept zu skizzieren, welches dem Studenten und der Komplexität der Übersetzungssituation gerecht wird. Leitgedanke dabei ist, dass der Student aus der Evaluation seiner Übersetzungsarbeiten, die er im Laufe des Semesters anfertigt, den größtmöglichen Nutzen ziehen können soll, und zwar auf eine Art, die ihm auch in seinem späteren Beruf als Übersetzer hilft. Ein- 
schränkend sei hier gesagt, dass „Diagnose“ und „Therapie“ ausgeklammert werden. Das heißt, beim vorliegenden Artikel geht es nicht darum herauszufinden, weshalb ein Übersetzungsstudent einen bestimmten Fehler gemacht hat und, von dieser Diagnose ausgehend, pädagogische Lösungen zur Fehlervermeidung zu suchen.

\section{VORANNAHMEN}

\subsection{Die Illusion der eindeutigen Entsprechung}

Beim Korrigieren von Rechenaufgaben kann die Lehrperson, da es genau eine richtige Lösung gibt, objektiv feststellen, ob das Resultat richtig ist oder nicht. Dem Korrektor von Übersetzungen hingegen ist dies verwehrt; denn zu einem Ausgangstext gibt es immer mehrere Übersetzungsmöglichkeiten. Da diese Behauptung von zentraler Bedeutung ist, verdient sie es, genauer betrachtet zu werden.

Prun (1997b: 33) zum Beispiel spricht von einer dreifachen Arbitrarität, die bei Übersetzungen zum Tragen komme. Eine davon ist „die Arbitrarität der Translationsnormen und -konventionen“" Weiter unten (ibid.: 34), stellt er der „grundsätzlichen Arbitrarität der Translationsnormen und -konvention“ die „ebenso grundsätzliche Arbitrarität des Skopos“ entgegen, und führt aus: „Aus dem Prinzip der Arbitrarität des Skopos folgt, dass grundsätzlich jede Relation zwischen AT und ZT möglich ist."

Hönig (1997: 32) seinerseits verweist darauf, dass die Symmetrie zwischen Ausgangs- und Zieltext eine „Illusion“ sei. Zur Begründung führt er unter anderem die Unmöglichkeit auf, die Bedeutung eines Textes sprachbefreit darzustellen. Er spricht in diesem Zusammenhang von der „Illusion der Interlingua“ (ibid.: 104) und weiter unten (ibid.: 112) von der selbstreferentiellen Bedeutung von Wörtern:

Wörter sind bereits das Resultat eines sprach- und kulturgebundenen Verarbeitungsprozesses "der" Realität; ihre Bedeutung lässt sich von ihrer Systemrelevanz nicht trennen. Bedeutung ist ein Knotenpunkt in einem Geflecht von Vernetzungen und Beziehungen, die kulturell-historisch gewachsen und individuell geprägt sind. Seine Bedeutung erhält der einzelne Knoten nicht durch das, was er abbildet — denn es gibt nichts, das außerhalb dieses Beziehungsnetzes beschreibbar wäre - , sondern durch die Relevanz, die er in diesem Beziehungsgeflecht für das individuelle Bewusstsein gewinnt.

Der Sinn ist demnach nicht im einzelnen Wort enthalten. Außerdem wird beim Verständnis auch das Weltwissen ${ }^{2}$ des Lesers eine Rolle spielen. Nun wäre es vermessen, in zwei Zeilen abhandeln zu wollen, wie Sinn entsteht. Für unsere Zwecke genügt es festzuhalten, dass kein Autor den Leser (und der Übersetzer ist in einer ersten Phase ebenfalls Leser) zwingen kann, seinen Text auf eine ganz bestimmte Weise aufzunehmen. Kurzum, weder auf der Ebene des Wortes noch auf derjenigen des Gesamttextes gibt es zwischen Ausgangs- und Zieltext vordefinierte und eindeutige Entsprechungen. Der Ausgangstext für sich genommen gibt keine eindeutigen Richtlinien - höchstens Hinweise - wie die Übersetzung auszusehen hat. Das heißt auch, die Lehrperson kann sich bei der Evaluation einer Übersetzung nicht nach einer Modellübersetzung richten, die eindeutig aus dem Ausgangstext hervorgehen und welche die einzig richtige Lösung darstellen würde. 


\subsection{Bestimmung der Funktion als Ausweg?}

Nach Katharina Reiss (1971: 32) muss bei der Analyse der Übersetzung untersucht werden, „welche Funktion die Sprache im vorliegenden Text jeweils ausübt“. Mit der Zuordnung des Ausgangstextes in einen der von ihr vorgeschlagenen Texttypen (inhalts-, form- und appellbetonter Text) wird die Funktion des Ausgangstextes näher bestimmt. Die Funktion des Zieltextes aber wird dadurch nicht quasi automatisch mitdefiniert. Sicherlich hilft eine solche Analyse, den Ausgangstext als Ganzes besser zu erfassen. Und in vielen Fällen wird mit dem Zieltext eine ähnliche Absicht verfolgt werden, wie sie mit dem Original verfolgt wurde. Der Übersetzer kann sich so vom Ausgangs- und vom Zieltext ein Gesamtbild machen, welches ihn bei Entscheidungen auf der Mikroebene (Wort, Satz) leiten kann. Aber es sind Situationen vorstellbar, in welchen der Auftraggeber mit der Übersetzung ein Zielpublikum anvisiert, welches sich vom Zielpublikum des Ausgangstextes grundsätzlich unterscheidet. Ein weiteres Problem eines solchen Ansatzes ist die Subjektivität. Beim Festlegen der Funktion des Originals ist ein gewisser Grad an Objektivität sicher erreichbar, doch lässt sich die Subjektivität Seitens des Übersetzers und des Revisors nicht gänzlich vermeiden, wie auch Adab (1998: 127ff) betont. Sie bedauert, dass der Kontext eines zu übersetzenden Textes im traditionellen Unterricht zu kurz komme. So könne beim Studenten der Eindruck entstehen, das Übersetzen bestehe einzig im Finden von äquivalenten Spracheinheiten. Folgerichtig schlägt sie, ähnlich wie Reiss, vor, dass bei der Evaluation (im Rahmen des universitären Unterrichts) bei der Beurteilung der Übersetzung von der Lehrperson entschieden werden müsse, ob die Übersetzung funktioniere, d.h. ob die Übersetzung texttypen- und zielgerecht sei. Allerdings räumt sie weiter unten ein, dass eine solche Beurteilung nie ganz objektiv sein könne. ${ }^{3}$ Es müsste also eine Möglichkeit gefunden werden, um den Grad an Objektivität zu erhöhen.

\subsection{Bilanz}

Da es keine absoluten Kriterien für das Beurteilen von Übersetzungen gibt, die in jedem Fall Gültigkeit hätten, da dem Übersetzer und dem Revisor eine gewisse Subjektivität eingeräumt werden muss (Brunette, 1996: 26f), und die Analyse des Ausgangstextes zwar notwendig, jedoch nicht hinreichend ist, stellt sich die Frage, wie die Qualität einer Übersetzung evaluiert werden soll. Im Rahmen dieses Artikels interessiert, wie bereits erwähnt, insbesondere die Evaluation in der Unterrichtssituation. Eine scharfe Trennung zwischen beruflichem und universitärem Umfeld wird jedoch nicht immer möglich sein, da die Praxisnähe ebenfalls ein Anliegen ist.

\section{POSTULAT}

Der Ansatz für die Evaluation von Übersetzungen, der hier verteidigt werden soll, besteht darin, ein Lastenheft zu erstellen, in welchem vor dem Übersetzen festgehalten wird, welche Punkte die Übersetzung erfüllen muss, damit sie akzeptiert werden kann. Mit anderen Worten, es soll jeweils ein Leistungsverzeichnis in schriftlicher Form erarbeitet werden, in welchem die Kriterien aufgeführt werden, denen die anvisierte Übersetzung zu genügen hat. ${ }^{4}$ Die Überlegung ist folgende: Wird die Übersetzung an 
vorher bestimmten Qualitätsmaßstäben gemessen, kann der Objektivitätsgrad erhöht werden. Ob es nun textinhärente Parameter sind, die durch die Analyse des Ausgangstextes gewonnen wurden und dann expliziert worden sind, oder ob es extralinguale Parameter sind, die von der Lehrperson - die sozusagen als Auftraggeber auftritt arbiträr bestimmt wurden, der Übersetzer (der Student) braucht nicht zu spekulieren, sondern kann sich nach ihm bekannten Kriterien richten - und der Revisor (die Lehrperson) kann sich auf den vorher erstellten Kriterienkatalog berufen, um seine Korrekturen zu erklären und allenfalls zu rechtfertigen: Argumente wie „es tönt besser“ oder „es gefällt mir besser“ werden so vermieden. Hier könnte der Einwand erhoben werden, dass sich die Übersetzung an das Original zu halten habe, und dass folglich nicht willkürlich Kriterien aufgestellt werden dürften. Doch sei daran erinnert, dass der Zieltext nicht eindeutig durch den Ausgangstext festlegt wird: „Die grundsätzliche Arbitrarität des Skopos erlaubt es, sowohl hinsichtlich der Beziehung zwischen Ausgangstext und Zieltext als auch hinsichtlich der Qualität der zielsprachlichen Ausprägung des Translats relativ leicht operationalisierbare Maßstäbe anzulegen.“ (Prun, 1997a: 116) Es besteht also ein großer Spielraum beim Festlegen der Kriterien - wichtig ist, dass sie dem Studenten von Beginn an bekannt sind. Welche Kriterien ins Lastenheft aufgenommen werden sollen, könnte allein von der Lehrperson bestimmt werden. Doch wäre es aus pädagogischer Sicht wohl zu begrüßen, wenn die Studenten bei der Erarbeitung zumindest eines Teils der Maßstäbe mitwirkten; denn so werden die Studenten angehalten, sich mit der Frage auseinander zu setzen, welches Ziel mit der Übersetzung, die sie anfertigen sollen, verfolgt wird und welche Bedingungen erfüllt sein müssen, damit die Übersetzung ihre intendierte Funktion erfüllen kann. Sie müssen sich zudem über das Umfeld (Publikation, Zielpublikum usw.) klar werden. So könnte dieser Kriterienkatalog während des Unterrichts gemeinsam erstellt, schriftlich festgehalten und dann an alle verteilt werden, bevor die Studenten mit der eigentlichen Übersetzung beginnen. Natürlich verpflichtet sich die Lehrperson, die Übersetzung anhand dieses Katalogs zu beurteilen. Im Anhang ist ein Beispiel eines möglichen Lastenhefts abgebildet. Dieses Beispiel dient alleine der Illustration und erhebt keinen Anspruch auf Vollständigkeit, da der Kriterienkatalog für jede Übersetzung neu ausgehandelt werden muß.

In einem nächsten Schritt soll etwas ausführlicher auf die Maßstäbe eingegangen werden, die in ein solches Lastenheft aufgenommen werden könnten. Dann wird es darum gehen, das Postulat zu begründen.

\section{MÖGLICHE MASSSTÄBE}

Prun (1997a: 116) gibt zu bedenken, dass es „unökonomisch [wäre], den Skopos in jedem konkreten Einzelfall neu aushandeln, festlegen und beschreiben zu wollen.“ Dies ist sicher nicht von der Hand zu weisen, insbesondere in einem professionellen, vom Streben nach Wirtschaftlichkeit geprägten Umfeld. In einem universitären Rahmen ist es indes zu begrüßen, wenn sowenig wie möglich stillschweigend vorausgesetzt wird, da sich der Student auch die impliziten Anforderungen an eine Übersetzung aneignen muss. Das würde heißen, dass im Rahmen des Unterrichts selbst Qualitätsparameter ins Lastenheft aufgenommen werden, die, wie zum Beispiel die sprachliche Korrektheit (siehe weiter unten), üblicherweise als selbstverständlich und deshalb als nicht erwähnenswert gelten. 
Im nächsten Abschnitt sollen einige mögliche Qualitätsmaßstäbe kommentiert werden, wobei diese Liste nur einen Ausschnitt aller möglichen Kriterien darstellt. Für eine umfassende Übersicht sei hier auf Brunette (1996: 29ff) und auf die „Systematik der translatorischen Kategorien“ von Stolze (1999: 240ff) verwiesen.

\subsection{Funktion der Übersetzung}

Grundlage für die Bestimmung der Funktion, welche die Übersetzung erfüllen soll, ist sicherlich die Analyse des Ausgangstextes, wie sie beispielsweise Reiss (1971) vorschlägt. Die Qualitätsmaßstäbe, die aus der Analyse gewonnen werden, können als textinhärente Maßstäbe bezeichnet werden. Doch wie bereits weiter oben argumentiert wurde, muss die gewollte Funktion des Zieltextes nicht mit derjenigen des Ausgangstextes übereinstimmen. So ist es denkbar, dass die Übersetzung eines Geschäftsbriefs „nur“ als internes Memo gedacht ist, welches Mitarbeiter, die der Fremdsprache nicht mächtig sind, knapp über den Inhalt des Briefes informieren soll. Außerdem lässt sich auch die Funktion des Ausgangstextes nicht immer vollständig und einzig aus dem Text erschließen: In vielen Fällen wird es nötig sein, dass die Lehrperson extralinguale Angaben wie Ort, Datum und Art der Publikation angibt. Doch sollte nicht nur die Publikation des Ausgangstextes situiert, sondern auch das Zielpublikum definiert werden. Mit der Aufnahme der Funktion der Übersetzung ins Lastenheft weiß der Student, wohin die Reise gehen soll. Andererseits - indem die Lehrperson Hintergrundsinformationen zum Ausgangstext gibt und explizite Vorgaben macht, so wie es ein Auftraggeber tun würde (oder zumindest tun sollte!) wird dem Studenten bewusst gemacht, dass es keine Übersetzung an und für sich gibt und dass eine Übersetzung immer orts-, zeit- und zielgebunden ist. Dies ist um so wichtiger für die Ausbildung des Studenten hin zum fachlich qualifizierten Übersetzer, als einer der Unterschiede zwischen dem Laienübersetzer und dem Experten gerade darin besteht, dass der Laie die Tendenz hat, weder die Zielsituation noch den tatsächlichen Auftrag zu berücksichtigen (Risku, 1998: 78f).

\subsection{Kohärenz}

Es kann nicht genug betont werden, dass der Zieltext eine in sich geschlossene Einheit bilden muss; denn es ist ein Leichtes, ob dem Übersetzen den Text in seiner Gesamtheit aus den Augen zu verlieren. Nach Stolze (1999: 243) gehört die Kohärenz in die Kategorie „Thematik“:

Hier ist oft auch die Frage nach einem dominanten Wortfeld, einer Isotopieebene im Text der Schlüssel für ein adäquates Verständnis des Ausgangstextes, insbesondere bei geisteswissenschaftlichen Textvorkommen. Andererseits stellt dies auch ein Kriterium der Textkohärenz in der Zielsprache dar, welche vom Auftraggeber als Plausibilität erwartet wird. Oft ist ja zu beobachten, dass Übersetzungen punktuell gesehen durchaus nicht falsch sind, dass aber der in Wortfeldern indizierte Gesamtzusammenhang völlig übersehen wurde.

Da die Kohärenz des Zieltext wichtig ist, und diesbezügliche Mängel im Ausgangstext keine Entschuldigung für schlecht geschrieben Übersetzungen darstellen, wird vorgeschlagen, dieses Kriterium ebenfalls zu berücksichtigen. 


\subsection{Sprachliche Korrektheit}

Beim Evaluationskriterium „sprachliche Korrektheit“ scheint der Konsens am größten zu sein. Immerhin gibt es Referenzwerke wie Le Petit Robert oder den Duden, die festsetzen, welche orthographischen und grammatikalischen Regeln Geltung haben. Korrekturen von Rechtschreibe- oder Interpunktionsfehlern scheinen Übersetzungsstudenten ohne weiteres zu akzeptieren, während sie der Meinung sind, bei der Korrektur von Stil sei Vorsicht am Platz, da Stil auch eine Frage von persönlichem Geschmack sei. Doch auch wenn die Bewertung der sprachlichen Korrektheit relativ unproblematisch ist, und die sprachliche Korrektheit wohl in erster Linie ein impliziter Qualitätsmaßstab ist (sowohl im Unterricht als auch in der Berufssituation) sollte die sprachliche Korrektheit beim Bestimmen der Evaluationskriterien zur Sprache kommen, ist es doch denkbar, dass gewisse Aspekte der Rechtschreibung explizit vorgegeben werden müssen. So ist eventuell festzulegen, für welche Region in der Welt die Übersetzung bestimmt ist (z.B. britisches vs. amerikanisches Englisch). In anderen Fällen wird bestimmt, wie die Orthographie von gewissen Fachtermini zu handhaben ist (Antispasmodika vs. Antispasmodica). In der Unterrichtssituation sind solche Unterscheidungen kaum relevant, doch ist es sicher sinnvoll, dass der Student und angehende Übersetzer lernt, mit solchen Style Sheets umzugehen und sich der Problematik bewusst wird.

\subsection{Vollständigkeit}

In den meisten Fällen wird implizit davon ausgegangen werden, dass die Übersetzung vollständig zu sein hat. Vereinfacht gesagt darf im Zieltext nichts fehlen, was im Ausgangstext vorkommt. Das scheint einleuchtend und selbstredend. Trotzdem sollte auch dieser Punkt in den Katalog der zu erfüllenden Kriterien aufgenommen werden, ist dies doch eine Gelegenheit darüber zu diskutieren, was „Vollständigkeit“ genau meint. Vielleicht wird der Übersetzer eine Erklärung, die im Original nicht steht, einführen wollen, weil er der Ansicht ist, dass sie für das Verständnis des Zielpublikum nötig sei. Oder der Übersetzer entscheidet sich, eine Straffung vorzunehmen. Wurde vor dem Übersetzen im Unterricht abgeklärt, ob und in welchem Fall solche Eingriffe gerechtfertigt oder gar wünschenswert sind, und wurde dieser Punkt zwischen Lehrperson und Student ausdrücklich festgelegt, besteht auch in diesem Punkt Klarheit.

\section{VORTEILE}

In diesem Abschnitt sollen die wichtigsten Argumente zusammengefasst werden, die dafür sprechen, im Unterricht mit einem Lastenheft zu arbeiten.

\subsection{Synchronie}

Dank der Evaluation anhand eines Lastenheftes wird dem Studenten bewusst gemacht, dass es die Übersetzung, die in jedem Fall die beste wäre, nicht gibt: Die Übersetzung ist nicht nur Momentaufnahme eines spezifischen Zeitpunktes in der sprachlichen Entwicklungsgeschichte einer Gemeinschaft; ${ }^{5}$ sie ist zudem auftragsspezifisch. 


\subsection{Transparenz und Vertrauen}

Indem vor der Übersetzung ein Lastenheft erarbeitet wird und gewissermaßen die Spielregeln vorher bekannt gemacht werden, wird (zumindest ein Stück weit) vermieden, dass der Student das Gefühl bekommt, die Arbeiten würden gemäss den persönlichen Vorlieben der Lehrperson evaluiert. Und wenn die Arbeit besprochen wird, kann sich die Lehrperson nicht auf ihre soziale Position berufen, sondern muss sich auf die vorher bestimmten Kriterien stützen, was es dem Studenten einfacher macht, Kritiken zu akzeptieren. Das Arbeiten mit expliziten Qualitätsanforderungen schafft Transparenz und Vertrauen.

\subsection{Vermittlung}

Nimmt man explizit deklarierte Vorlagen als Grundlage der Übersetzungsevaluation, ist es müßig, darüber zu streiten, ob eine Übersetzung frei oder wörtlich zu sein hat. In einem solchem Fall wurde nämlich zwischen den Handlungspartnern vorher definiert, ob bei der Übersetzung (dem Translat) die Einbettung in die Zielkultur oder die Hervorhebung der Unterschiede, die zwischen Ausgangs- und Zielkultur bestehen, im Vordergrund stehen soll. Ob die beiden Partner „Auftraggeber und Übersetzer“ oder „Lehrperson und Student“ heißen, spielt dabei keine Rolle. Vergessen wir nicht, dass der Übersetzer nie neutraler Vermittler von objektiven Informationen sein kann (auch bei der sogenannten wörtlichen Übersetzung nicht). Übersetzen ist ein kreativer Akt. Mit der Festlegung von Evaluationskriterien wird dies dem Übersetzer zugestanden, ohne dass auf der anderen Seite der Willkür Tür und Tor geöffnet würden, da eine Art Vertrag zwischen zwei verantwortlichen Partnern geschlossen wird. Mit anderen Worten, der Übersetzer wird nicht zur Maschine reduziert, die, ohne einen eigenen Beitrag zu leisten, bloß den Anweisungen des Ausgangstextes folgt. Gleichzeitig darf und muss der Übersetzer für seinen Text Verantwortung übernehmen.

\subsection{Vorbereitung}

Ein Auftraggeber kann die Qualität der Übersetzung zwar nicht direkt beeinflussen, aber doch ein Umfeld schaffen, welches zur Erhöhung der Qualität beiträgt. Dieser Tatsache trägt die DIN-Norm 2345 (Punkt 4.3 „Unterstützung durch den Auftraggeber“, Seite 8f) Rechnung: „Der Auftraggeber muß den Übersetzer so weit wie möglich und für den Auftrag sinnvoll durch die Bereitstellung einschlägiger Materialien und betrieblicher Informationen unterstützen; dazu kann folgendes gehören: a) Fachliteratur, b) Terminologielisten, c) Glossare, d) Paralleltexte, e) Hintergrundtexte, f) Betriebsbesichtigungen. Darüber hinaus muß der Auftraggeber dem Übersetzer kompetente Ansprechpartner benennen, die ihm für Rückfragen zur Verfügung stehen. "Durch das Arbeiteten mit Vorgaben wird der Student auf das Berufsleben vorbereitet. Entweder arbeitet er nach dem Studium für ein Unternehmen, das sich seiner Verantwortung bewusst ist und dem Übersetzer deshalb Hintergrundsmaterial, Leitfaden für die Schreibweise etc. zur Verfügung stellt. In diesem Fall wird der Übersetzer sich schnell in die Firmenkultur einleben, weil er diese Art zu arbeiten bereits kennt. Oder aber er arbeitet für einen Auftraggeber, welcher der Meinung ist, 
solche Vorkehrungen seien überflüssig, weil der Übersetzer „einfach übersetzen soll, was da steht“. Hier kann der Übersetzer als kompetenter und verantwortungsbewusster Partner auftreten, da er in der Lage ist, klar darzustellen, welche Voraussetzungen gegeben sein müssen, damit die Übersetzung qualitativ hochstehend sein kann.

\subsection{Denn sie wissen was sie tun}

Dadurch, dass Studenten lernen, mit Vorgaben umzugehen und vor allem solche im Unterricht zu erarbeiten, üben sie sich darin, ihr tun zu reflektieren. Hönig (1997: 19f) stellt dem Bild des Über-setzers (sic) — dessen Aufgabe darin besteht, „die Distanz zwischen den Ufern (Texten, Sprachen, Kulturen) sozusagen horizontal zu überwinden“ — dasjenige des Brückenbauers entgegen - dessen Aufgabe in der „Errichtung einer tragfähigen Konstruktion, auf die dann die horizontale Verbindungsstrecke aufgelegt werden kann", besteht:

[Der Unterschied] wird noch klarer, wenn wir zwei gegensätzliche Thesen für die Übersetzungsausbildung davon ableiten:

- Für die professionelle Ausübung der Übersetzerberufs braucht man ein Schiff (= Wörterbuch, Computer und Faxgerät) und sehr viel Übung. Also: Übersetzen lernt man durch Übersetzen.

- Es gibt Kenntnisse und Fertigkeiten, die nicht empirisch erworben werden können wie zum Beispiel ein Architekt Kenntnisse auf dem Gebiet der Statik, Materialkunde usw. durch ein Hochschulstudium erwerben muß. Also: Übersetzen lernt man durch den Erwerb übersetzerischer Kompetenz.

Das Arbeiten mit einem Lastenheft ist ganz im Sinne einer Unterrichtsform, die übersetzerischen Kompetenzen vermitteln will. Das Arbeiten mit einem Lastenheft kann für den Studenten eine Möglichkeit sein, sich auf praxisorientierte und konkrete Weise übersetzerische Kompetenzen anzueignen, da der Student zur Erarbeitung der Kriterien seinen Beitrag leisten und im Verlauf des übersetzerischen Prozesses seine Arbeit kritisch an eben diesen Maßstäben messen kann.

\section{ZUSAMMENFASSUNG}

Ausgangspunkt dieses Artikels war die Feststellung, dass es für einen Ausgangstext so viele Übersetzungen geben wird wie ihn Personen übersetzen. Außerdem ist der Begriff der Qualität in der Übersetzung nur sehr schwer zu fassen. Dies hat zur Folge hat, dass sich die Evaluation schwierig gestaltet. Der hier skizzierte Ansatz kann nicht alle Schwierigkeiten beseitigen. Wird aber mit klaren Leistungsvorgaben gearbeitet, kann zumindest ein gewisser Grad an Transparenz und Objektivität erreicht werden, auch wenn bei der Entscheidung, ob eine Übersetzung den Kriterien genügt, ein subjektives Element bleibt. Des Weiteren hat der Student auf diese Weise die Möglichkeit, eine kritische Distanz zum übersetzerischen Prozess und dessen Produkt zu wahren. Beides, erhöhte Objektivität und kritische Distanz, wird dadurch erreicht, dass die Bewertung der Übersetzung auf einer höheren Abstraktionsebene erfolgt. Aus diesen Gründen scheint es durchaus lohnend, diesen Ansatz weiterzuverfolgen. 


\section{ANMERKUNGEN}

1. Gemäß Prun sind die grundsätzlich arbiträren Translationsnormen und -konventionen in ein Normensystem eingebunden, welches er als Translationskultur bezeichnet. Diese Translationskultur wiederum ist Ausdruck der herrschenden Machtstrukturen und Ideologien.

2. Gesamtheit des erworbenen Wissens als allgemeiner Wissensfundus eines Menschen. (Delisle 1999: 413)

3. Ainsi, pour ce qui est de l'évaluation de la traduction dans un cours universitaire, le succès d'une traduction devra être mesuré au degré d'adéquation à la fonction du texte, au respect de sa finalité. On évaluera les erreurs dans le contexte plus large du message global, de la structure du texte et de l'effet sur le lecteur. Il faut reconnaître que l'objectivité dans ce genre d'évaluation ne saurait être absolue, étant donné la nature subjective et indéterminée du concept de norme et de texte prototypique pour chaque fonction, chaque genre de texte. (Adab, 1998: 130)

4. Hier soll das Arbeiten mit einem Lastenheft im Rahmen des Unterrichts verteidigt werden. Für die Evaluation einer Übersetzung gemäß eines Lastenheftes im professionellen Umfeld sei auf Larose (1998) verwiesen.

5. Or, une des caractéristiques de la traduction est sa nature fugitive: une traduction correspond à un moment précis (synchronie) de la situation et de l'histoire linguistiques d'un groupe, qu'elle cristallise et fige en même temps, puisque l'on dit d'un texte traduit depuis un certain temps qu'il «date». (Gémar 1995: 33)

\section{BIBLIOGRAPHIE}

\section{Artikel und Bücher}

ADAB, B. J. (1998): «Évaluer les traductions en fonction de la finalité des textes», Enseignement de la traduction et traduction dans l'enseignement (J. Delisle et H. Lee-Jahnke, dir.), Ottawa, Presses de l'Université d'Ottawa, p. 127-133.

Brunette, L. (1996): «Quelle objectivité pour la révision?», Traduire, 168, p. 25-33.

Delisle, J., H. Lee-Jahnke et M. C. Cormier (1999): Terminologie de la traduction, Amsterdam, John Benjamins.

GÉmar, J.-C. (1995): Traduire ou l'art d'interpréter, Québec, Presses de l'Université de Québec. HönIG, H. G. (1997): Konstruktives Übersetzen. 2., durchges. Aufl. Tübingen: Stauffenburg

Larose, R. (1998): «Méthodologie de l'évaluation des traductions», Meta, 43-2, p. 163-184

PRUN, E. (1997a): „Translationskultur (Versuch einer konstruktiven Kritik des translatorischen Handelns)“. In: TEXTconTEXT = NF 1. S. 99-127

— (1997b): „Versuch einer Skopostyplogie“. In: Grbi?, Nadja und Wolf, Michaela (Hrsg.): Text Kultur - Kommunikation. Tübingen: Stauffenburg

ReIss, K. (1971): Möglichkeiten und Grenzen der Übersetzungskritik: Kategorien und Kriterien für eine sachgerechte Beurteilung von Übersetzungen. München: Max Hueber

Risku, H. (1998): Translatorische Kompetenz: kognitive Grundlagen des Übersetzens als Expertentätigkeit. Tübingen: Stauffenburg

Stolze, R. (1999): Die Fachübersetzung: eine Einführung. Tübingen: Narr.

\section{Normen}

DIN 2345: 1998-04: Übersetzungsaufträge 


\section{ANHANG}

\section{Beispiel eines Lastenhefts}

Evaluation des Zieltextes (Kommentar)

Ausgangstext (AT)

\begin{tabular}{|l|l|}
\hline Sprache & Englisch \\
Quelle & $\begin{array}{l}\text { Geldart, William (1995): Introduction to } \\
\text { English Law. Oxford University Press }\end{array}$ \\
\hline
\end{tabular}

Zieltext (ZT)

\begin{tabular}{|c|c|}
\hline Sprache & Deutsch \\
\hline Funktion & $\begin{array}{l}\text { Die Übersetzung soll als Lehrbuch } \\
\text { dienen (kein Funktionswechsel von AT } \\
\text { zu ZT). }\end{array}$ \\
\hline Zielpublikum & $\begin{array}{l}\text { Deutschsprachige Rechtsstudenten, die } \\
\text { ins englische Rechtssystem eingeführt } \\
\text { werden sollen. }\end{array}$ \\
\hline Sprachliche Korrektheit & $\begin{array}{l}\text { keine Verstöße gegen Grammatik, } \\
\text { Orthographie usw. }\end{array}$ \\
\hline Aussage & $\begin{array}{l}\text { keine punktuellen Sinnfehler } \\
\text { keine Sinnfehler bezüglich des Kontextes } \\
\text { (Isotopie-Ebene) }\end{array}$ \\
\hline Kohärenz & $\begin{array}{l}\text { ZT muss in sich stimmig und ohne AT } \\
\text { verständlich sein }\end{array}$ \\
\hline Stil / Register & $\begin{array}{l}\text { nüchtern-sachlich } \\
\text { an das Zielpublikum angepasst } \\
\text { unpersönlich, keine direkte Anrede des } \\
\text { Lesers (anders als im englischen } \\
\text { Original) } \\
\text { Die Exaktheit des Begriffs ist wichtiger } \\
\text { als das Vermeiden von Wort- } \\
\text { wiederholungen }\end{array}$ \\
\hline $\begin{array}{l}\text { Paralleltext (fürs Verständnis } \\
\text { und als Stilvorlage) }\end{array}$ & $\begin{array}{l}\text { Bernstorff, Graf von (1996): Einführung } \\
\text { in das englische Recht. } \\
\text { München: Beck }\end{array}$ \\
\hline Kulturspezifische Ausdrücke & $\begin{array}{l}\text { Steht in der Zielsprache kein Äquivalent } \\
\text { zur Verfügung (= Term, das ein Konzept } \\
\text { bezeichnet, das innerhalb der jeweiligen } \\
\text { Rechtssysteme die gleiche Bedeutung } \\
\text { hat), soll der ausgangssprachliche } \\
\text { Ausdruck (kursiv) beibehalten und in } \\
\text { einem Nebensatz oder in einer Klammer } \\
\text { (keine Fußnoten) erläutert werden. }\end{array}$ \\
\hline
\end{tabular}

